\title{
Mineralization of aromatic compounds by brown-rot basidiomycetes - mechanisms involved in initial attack on the aromatic ring
}

\author{
Fumika Kamada, Suzuna Abe, Nobuhiro Hiratsuka, Hiroyuki Wariishi \\ and Hiroo Tanaka
}

Faculty of Agriculture, Kyushu University, 6-10-1, Hakozaki, Higashi-ku, Fukuoka 812-8581, Japan
Author for correspondence: Hiroyuki Wariishi. Tel/Fax: +8192642 2993. e-mail: hirowari@agr.kyushu-u.ac.jp

Benzaldehyde and its metabolic intermediates were effectively degraded by the brown-rot basidiomycetes Tyromyces palustris and Gloeophyllum trabeum. The pathway of benzaldehyde degradation was elucidated by the identification of fungal metabolites produced upon the addition of benzaldehyde and its metabolic intermediates. The oxidation and reduction occurred simultaneously, forming benzyl alcohol and benzoic acid as major products. Hydroxylation reactions, which seemed to be a key step, occurred on benzaldehyde and benzoic acid, but not on benzyl alcohol, to form corresponding 4-hydroxyl and 3,4-dihydroxyl derivatives. 1-Formyl derivatives were oxidized to 1-carboxyl derivatives at several metabolic stages. All of these reactions resulted in the formation of 3,4-dihydroxybenzoic acid. This was further metabolized via the decarboxylation reaction to yield 1,2,4trihydroxybenzene, which may be susceptible to the ring-fission reaction. Ring$\mathrm{U}-{ }^{14} \mathrm{C}$-labelled benzaldehyde and benzoic acid were effectively mineralized, clearly indicating that the brown-rot basidiomycetes are capable of metabolizing certain aromatic compounds to $\mathrm{CO}_{2}$ and $\mathrm{H}_{2} \mathrm{O}$, despite the fact that brown-rot fungi cannot degrade polymeric lignin. Inhibitor experiments, using hydroxyl radical scavengers, catalase and cytochrome $\mathbf{P 4 5 0}$ inhibitors, strongly suggested that the aromatic hydroxylation reactions found in the brown-rot fungi are catalysed by intracellular enzyme(s), but not by Fenton-reactionderived hydroxyl radicals.

Keywords: brown-rot fungus, benzaldehyde, hydroxylation, lignin, metabolism

\section{INTRODUCTION}

Wood-rotting basidiomycetes have been categorized into two groups, white-rot and brown-rot fungi, according to their ability to remove lignin from wood components during decay. The correlation between ligninolytic activity and Bavendamm's reaction has been known for 70 years (Bavendamm, 1928). White-rot fungi, which are capable of degrading lignin, produce a coloured zone around mycelia on agar plates containing tannin or other phenolic substrates. The colourization is caused by extracellularly secreted phenoloxidases. Brown-rot fungi, which cannot degrade lignin, do not cause this colourization (Bavendamm, 1928; Kirk \& Kelman, 1965; Sundman \& Näse, 1971). However, the

Abbreviations: $\mathrm{HN}$, high nitrogen; LN, low nitrogen. brown-rot basidiomycetes are known to be responsible for severe decay of woody cell walls (Zabel \& Morell, 1992). Thus, brown-rot fungi effectively digest cellulosic components without removing lignin, which retards microbial attacks on woody carbohydrates (Crawford, 1981; Eriksson et al., 1990). However, the residual lignin in brown-rotted wood is modified, giving a decrease of methoxyl-content and an introduction of carboxyl and phenolic hydroxyl groups (Brown et al., 1968; Kirk, 1975).

The involvement of a low-molecular-mass one-electron oxidant, such as the hydroxyl radical and Fenton reagent, has been suggested for brown-rot-type wood decay (Backa et al., 1992; Chandhoke et al., 1992; Goodell et al., 1997; Hirano et al., 1995, 1997; Hyde \& Wood, 1997; Koenigs, 1975). Recently, an effective cleavage of polyether was shown to be catalysed by the 
brown-rot basidiomycete Gloeophyllum trabeum via extracellular one-electron oxidation caused by hydroquinone-driven Fenton reaction (Kerem et al., 1998, 1999). Besides a partial modification of lignin, the degradation of lignin model compounds by brown-rot fungi has been reported (Enoki et al., 1985), but neither the detail of reaction pathways nor the fate of aromatic components has been elucidated. If the Fenton type of reaction is involved in the brown-rot fungal metabolism, the effect of the hydroxyl radical on the aromatic moiety is of great interest.

In this study, benzaldehyde was utilized as the aromatic substrate for the brown-rot basidiomycetes Tyromyces palustris and G. trabeum. Product analysis revealed the occurrence of benzylic oxidation and reduction. A sitespecific hydroxylation of benzaldehyde and benzoic acid was also found to be catalysed by these brown-rot fungi. Furthermore, no inhibition was observed for the hydroxylation reactions by either mannitol or thiourea, well-known hydroxyl radical scavengers. Hydroxylated intermediates were further metabolized rapidly without the occurrence of the phenoloxidase-derived radical coupling reactions which have been observed for whiterot fungi. Finally, the effective mineralization of aromatic components by the brown-rot fungi, proof of the occurrence of ring-fission reactions, was confirmed using radioactive substrates.

\section{METHODS}

Organisms. The brown-rot basidiomycetes Tyromyces palustris (IFO 30339) and Gloeophyllum trabeum (IFO 6268) were grown for metabolic studies from hyphal inocula at $30^{\circ} \mathrm{C}$ in stationary cultures $(10 \mathrm{ml}$ medium in $100 \mathrm{ml}$ flasks). The medium used in this study was as previously described, with $1 \%$ glucose and either 1.2 or $12 \mathrm{mM}$ ammonium tartrate [lownitrogen (LN) and high-nitrogen $(\mathrm{HN})$ culture conditions, respectively] as the carbon and nitrogen source, at $\mathrm{pH} 6.0$ (Kirk et al., 1978). After a period of incubation under air (5 days for T. palustris, 7 days for G. trabeum), flasks were fitted with ports and were purged daily with either oxygen or air (15 min per day).

Chemicals. Benzyl alcohol, benzaldehyde, benzoic acid, 4-hydroxybenzaldehyde, 4-hydroxybenzoic acid, 3,4-dihydroxybenzaldehyde (protocatechualdehyde), 3,4-dihydroxybenzoic (protocatechuic) acid, 1,4-dihydroxybenzene (hydroquinone) and 1,2,4-trihydroxybenzene were obtained from Wako Pure Chemicals. Methyl 4-hydroxybenzoate was prepared from 4-hydroxybenzoic acid refluxed in dry methanol in the presence of catalytic $\mathrm{H}_{2} \mathrm{SO}_{4}$. All substrates were recrystallized or purified using a silica gel column or preparative HPLC before use. Hydrogen peroxide $(30 \%)$, mannitol, thiourea and piperonyl butoxide were purchased from Wako Pure Chemicals. 1-Aminobenzotriazole was obtained from TCI. All other chemicals were reagent grade. Deionized water was obtained from Milli Q System (Millipore).

Ring-U- ${ }^{14} \mathrm{C}-$ labelled benzaldehyde was purchased from Muromachi Chemical Engineering Co.; it was oxidized by $\mathrm{KMnO}_{4}$ to obtain ring-U- ${ }^{14} \mathrm{C}$-labelled benzoic acid. Labelled substrates were purified by preparative TLC (ethyl acetate/hexane, $1: 1$, $\mathrm{v} / \mathrm{v}$ ) before use.

Enzymes. Laccase, manganese peroxidase and lignin peroxidase activities in culture medium were measured as 2,6- dimethoxyphenol oxidation in the absence of $\mathrm{H}_{2} \mathrm{O}_{2}$ and $\mathrm{Mn}^{\mathrm{II}}$, $\mathrm{Mn}^{\mathrm{III}}$-malonate complex formation, and veratryl alcohol oxidation activities, respectively, as previously described (Hiratsuka et al., 2001; Ichinose et al., 1999; Kirk \& Farrell, 1987; Wariishi et al., 1992). Catalase was commercially obtained from Sigma.

Metabolic reactions. After a period of incubation as described above, the substrates were added to cultures to a final concentration of $0.5 \mathrm{mM}$. Metabolic products were analysed either by HPLC after homogenization, centrifugation $(2000 \mathrm{~g}$; $5 \mathrm{~min})$, and filtration $(0.45 \mu \mathrm{m})$, or by GC-MS after extraction with ethyl acetate at $\mathrm{pH} 2$, drying over $\mathrm{Na}_{2} \mathrm{SO}_{4}$, evaporation under $\mathrm{N}_{2}$, and derivatization using BSTFA/pyridine $(2: 1, \mathrm{v} / \mathrm{v})$ as previously described (Valli et al., 1992; Wariishi et al., 1989).

Fenton reaction was performed in $500 \mathrm{mM}$ acetate buffer $(\mathrm{pH}$ $4 \cdot 5$ ) containing $5 \mu \mathrm{M} \mathrm{FeSO}_{4}, 0 \cdot 01 \% \mathrm{H}_{2} \mathrm{O}_{2}$ and $0.5 \mathrm{mM}$ benzoic acid. The reaction was initiated by the addition of $\mathrm{H}_{2} \mathrm{O}_{2}$, then performed at room temperature in the dark for $24 \mathrm{~h}$.

Effect of inhibitors. Mannitol and thiourea were added to the cultures to final concentrations of 50 and $25 \mathrm{mM}$, respectively, $5 \mathrm{~min}$ prior to the addition of benzoic acid. Catalase (5900 U) was added to the cultures immediately prior to the addition of benzoic acid. Piperonyl butoxide and 1-aminobenzotriazole (final concentration $2.5 \mathrm{mM}$ ) were added to the cultures 30-90 min prior to the addition of benzoic acid (Eilers et al., 1999; Hiratsuka et al., 2001; Ichinose et al., 1999). The quantitative analysis was performed as described above.

These inhibitors were added immediately prior to the addition of $\mathrm{H}_{2} \mathrm{O}_{2}$ when examined in the Fenton reaction system. Products were analysed using HPLC as described above after centrifugation and filtration.

Mineralization of ${ }^{14} \mathrm{C}$-labelled substrates. Ring-U- ${ }^{14} \mathrm{C}$-labelled substrates (benzaldehyde or benzoic acid) were added to cultures $\left(10^{4}\right.$ d.p.m. per flask), which were purged with oxygen or air as described above. Evolved ${ }^{14} \mathrm{CO}_{2}$ was trapped in a basic scintillation fluid as previously described (Kirk, 1975; Valli \& Gold, 1991) and radioactivity was measured in a Beckman LS-6500 scintillation counter. The efficiency of ${ }^{14} \mathrm{CO}_{2}$ trapping after 10 min purging was greater than $98 \%$. The residual radioactivity in flasks was determined after mixing the medium with a Beckman Ready-Solv scintillation cocktail. The counting efficiency was monitored with an automatic external standard.

Chromatography and spectrometry. GC-MS was performed with a JMS-AM II 15 mass spectrometer (JEOL) at $70 \mathrm{eV}$ fitted with a Shimadzu GC-17A gas chromatograph and a fused silica column (30-m DB-5, J \& W Scientific). The GC temperature was programmed from 80 to $280{ }^{\circ} \mathrm{C}$ at $8{ }^{\circ} \mathrm{C}$ $\min ^{-1}$.

Products were also analysed by HPLC using an STR ODS-II column (Shimadzu) with a linear gradient from $20 \%$ acetonitrile in $0.05 \%$ phosphoric acid to $100 \%$ acetonitrile in $16 \mathrm{~min}$ (flow rate $1.0 \mathrm{ml} \mathrm{min}^{-1}$ ). A UV detector at 210,254 or $280 \mathrm{~nm}$ was used for monitoring the substrate and products. Products were identified by comparison of their retention times on GC and HPLC and of their mass fragmentation patterns with standards. Quantitative analysis was achieved by HPLC using calibration curves prepared with standards.

\section{RESULTS}

\section{Metabolism of benzaldehyde}

Products obtained from the metabolism of benzaldehyde by the two brown-rot fungi are shown in Fig. 1 and 


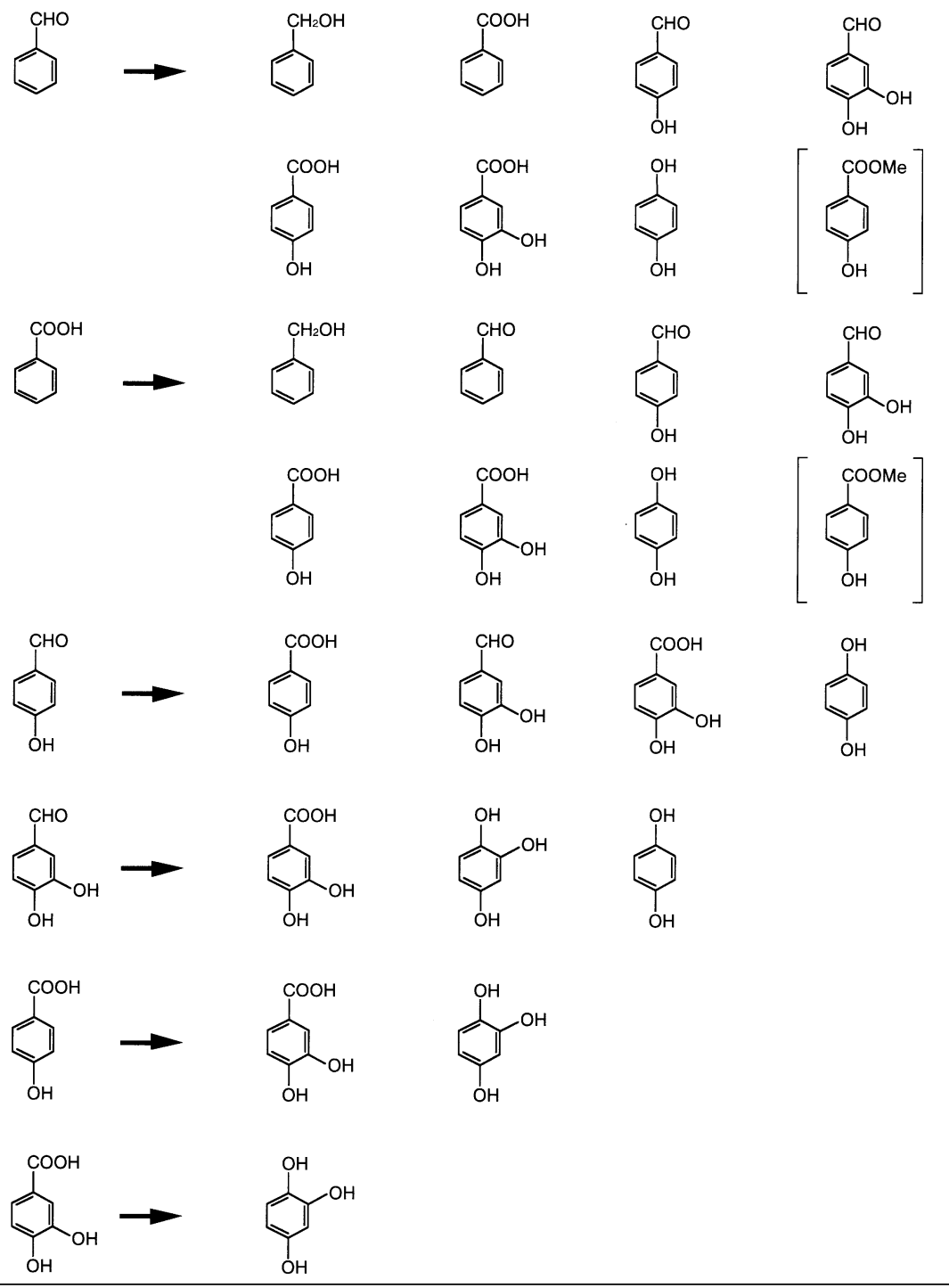

Fig. 1. Metabolites from benzaldehyde and its metabolic intermediates by $T$. palustris and G. trabeum. Products shown in brackets were found only in $T$. palustris cultures. Other products were confirmed in both fungal cultures. Fungal treatment was performed under air in $\mathrm{HN}$ conditions for $24 \mathrm{~h}$.

Table 1. Products obtained from the metabolism of benzaldehyde by the brown-rot fungi

\begin{tabular}{|lcc|}
\hline Substrate or metabolite & $\begin{array}{c}\text { GC retention } \\
\text { time }(\mathrm{min})\end{array}$ & Mass spectrum $\boldsymbol{m} / \boldsymbol{z}$ (relative intensity) \\
\hline Benzaldehyde & $3 \cdot 5$ & $106\left(\mathrm{M}^{+}, 100\right), 105(98 \cdot 8), 77(83 \cdot 4)$ \\
Benzyl alcohol (TMS*) & $8 \cdot 1$ & $180\left(\mathrm{M}^{+}, 7 \cdot 6\right), 165(68 \cdot 8), 135(84 \cdot 6), 91(100), 73(20 \cdot 5)$ \\
Benzoic acid (TMS) & $9 \cdot 8$ & $194\left(\mathrm{M}^{+}, 6 \cdot 4\right), 179(71 \cdot 3), 135(55 \cdot 6), 105(100), 77(60 \cdot 6)$ \\
4-Hydroxybenzaldehyde (TMS) & $12 \cdot 3$ & $194\left(\mathrm{M}^{+}, 67 \cdot 7\right), 193(9 \cdot 9), 179(100), 151(78 \cdot 6), 135(14 \cdot 9), 105(8 \cdot 3)$, \\
& & $89(30 \cdot 1), 73(36 \cdot 9)$ \\
4-Hydroxybenzoic acid (diTMS) & $16 \cdot 1$ & $282\left(\mathrm{M}^{+}, 26 \cdot 3\right), 267(100), 223(69 \cdot 1), 193(47 \cdot 1), 126(25 \cdot 6), 73(98 \cdot 6)$ \\
3,4-Dihydroxybenzaldehyde (diTMS) & $16 \cdot 0$ & $282\left(\mathrm{M}^{+}, 23 \cdot 7\right), 281(12 \cdot 3), 267(22 \cdot 7), 193(11 \cdot 1), 73(100)$ \\
3,4-Dihydroxybenzoic acid (triTMS) & $18 \cdot 7$ & $370\left(\mathrm{M}^{+}, 48 \cdot 7\right), 354(23 \cdot 2), 311(32 \cdot 2), 281(12 \cdot 7), 223(5 \cdot 9), 193(81 \cdot 5)$, \\
& & $73(100)$ \\
1,4-Dihydroxybenzene (diTMS) & $12 \cdot 7$ & $254\left(\mathrm{M}^{+}, 77 \cdot 8\right), 239(100), 223(5 \cdot 6), 112(26 \cdot 5), 73(69 \cdot 0)$ \\
Methyl 4-hydroxybenzoate (TMS) & $14 \cdot 1$ & $224\left(\mathrm{M}^{+}, 51 \cdot 9\right), 209(100), 193(29 \cdot 5), 177(21 \cdot 0), 149(20 \cdot 5), 135(52 \cdot 9)$, \\
& & $89(39 \cdot 6), 73(50 \cdot 8)$ \\
1,2,4-Trihydroxybenzene (triTMS) & $15 \cdot 8$ & $342\left(\mathrm{M}^{+}, 73 \cdot 8\right), 327(4 \cdot 8), 254(3 \cdot 1), 239(27 \cdot 8), 73(100)$ \\
\hline
\end{tabular}

* TMS, trimethylsilylated derivative. 


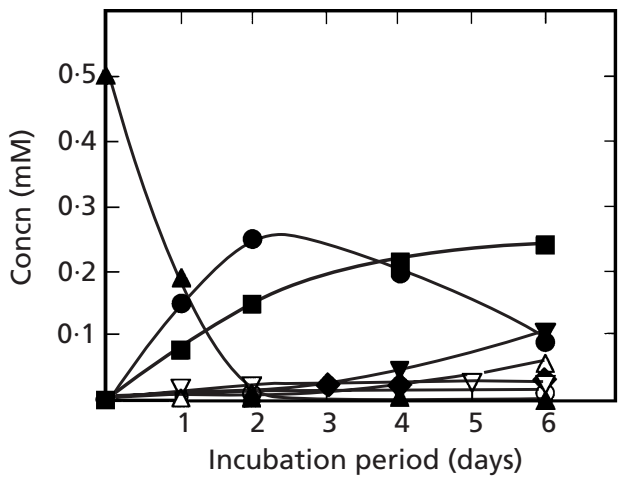

Fig. 2. Time-course for metabolism of benzaldehyde $(\boldsymbol{A})$ and its intermediates [benzoic acid (O), benzyl alcohol ( $\boldsymbol{\square})$, 4-hydroxybenzaldehyde $(\diamond)$, protocatechualdehyde $(\square)$, 4-hydroxybenzoic acid $(\boldsymbol{\nabla})$, protocatechuic acid (O), hydroquinone $(\triangle)$ and trihydroxybenzene $(\nabla)$ ] by $T$. palustris. Metabolic experiments were repeated five times. To better exhibit the quantitative relationship between the substrate and products, typical results are shown in this figure. The SD of each plot was less than $12 \%(n=5)$.

Table 1. Benzyl alcohol and benzoic acid were the major products. Five hydroxylated intermediates, 4-hydroxybenzaldehyde, 4-hydroxybenzoic acid, protocatechualdehyde, protocatechuic acid and hydroquinone, were also found as trace products. Methyl 4-hydroxybenzoate was found only in cultures of T. palustris (Fig. 1). No formation of quinones was observed. Although de novo synthesis of certain aromatic compounds by brown-rot fungi has been reported (Goodell et al., 1997; Paszczynski et al., 1999), the control cultures of T. palustris and G. trabeum exhibited no formation of any of the compounds shown in Fig. 1.

When benzoic acid was added to cultures of the brownrot fungi, products identical to those from benzaldehyde were observed (Fig. 1). 4-Hydroxybenzaldehyde was converted to protocatechualdehyde, 4-hydroxybenzoic acid, protocatechuic acid and hydroquinone. Protocatechualdehyde was converted to protocatechuic acid and trihydroxybenzene. 4-Hydroxybenzoic acid was metabolized to protocatechuic acid, hydroquinone and trihydroxybenzene. Protocatechuic acid was converted to trihydroxybenzene (Fig. 1). A methyl esterification reaction occurred on benzaldehyde and benzoic acid but not on 4-hydroxybenzaldehyde and 4-hydroxybenzoic acid (Fig. 1). Methyl 4-hydroxybenzoate was not further metabolized by T. palustris, showing that it is a deadend product.

\section{Time-course of metabolism}

To better understand the metabolic pathway, the timecourses of the disappearance of the substrate and the formation of products were monitored using HPLC. Fig. 2 shows the metabolism of benzaldehyde and its metabolic intermediates by $T$. palustris in $\mathrm{HN}$ cultures under air. During the metabolism of benzaldehyde,

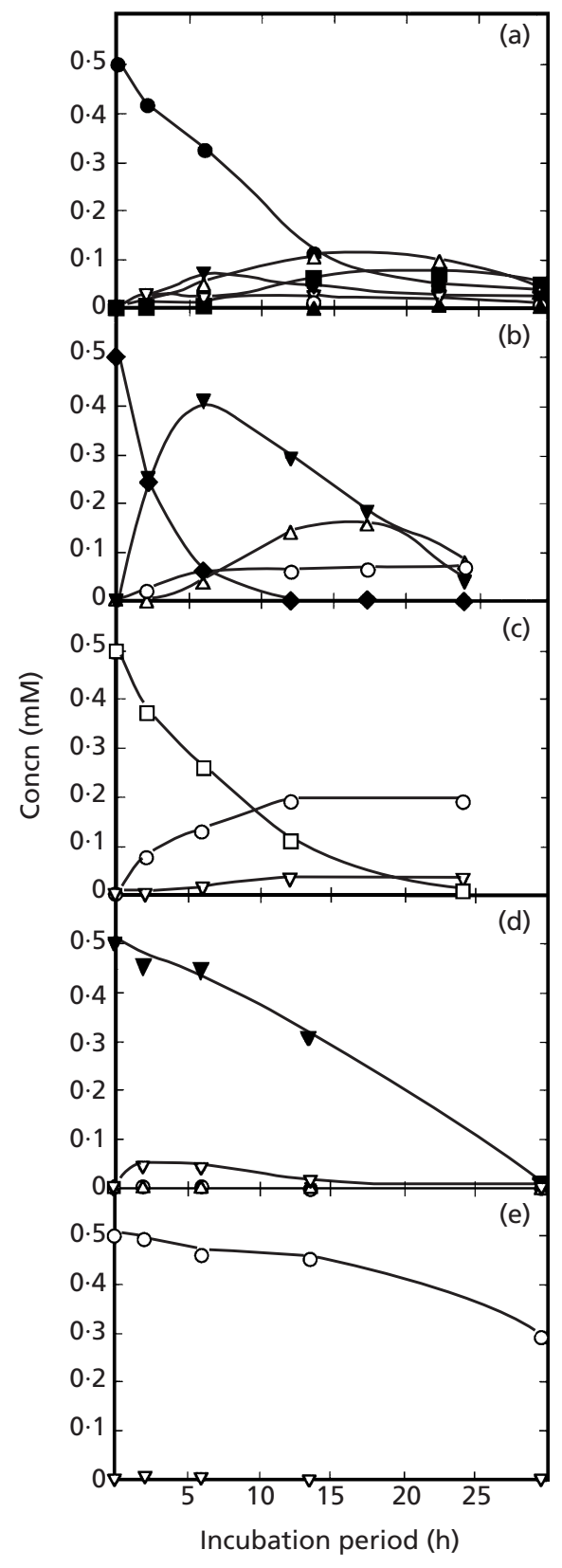

Fig. 3. Metabolic conversion by $T$. palustris of exogenously added intermediates of benzaldehyde metabolism. Starting substrates used were: (a) benzoic acid (O), (b) 4-hydroxybenzaldehyde ( $\bullet$ ), (c) protocatechualdehyde ( $\square$ ), (d) 4-hydroxybenzoic acid $(\boldsymbol{\nabla})$, and (e) protocatechuic acid (O). Other symbols for products and experimental conditions are the same as for Fig. 2.

benzoic acid was accumulated in the early stages of incubation and disappeared after day 2 , indicating that benzoic acid was a metabolic intermediate and was further converted to 4-hydroxybenzoic acid, protocatechuic acid, hydroquinone and trihydroxybenzene (Fig. 2). Since benzaldehyde was also directly hydroxylated to yield 4-hydroxybenzaldehyde and protocatechualdehyde (Fig. 1), those intermediates were also exogenously 


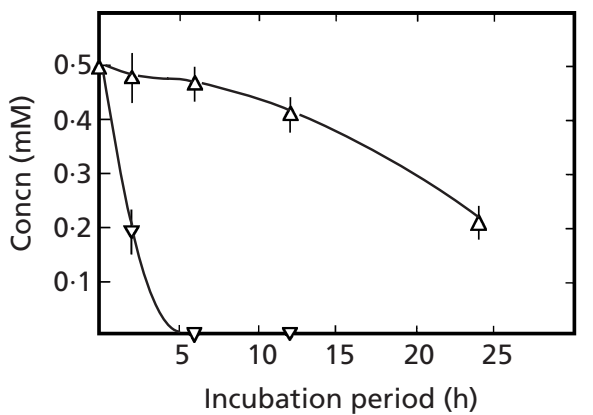

Fig. 4. Time-course for metabolism of hydroquinone $(\triangle)$ and trihydroxybenzene $(\nabla)$ by $T$. palustris. Each plot is the mean of the data obtained from five different cultures. The error bars show SD.

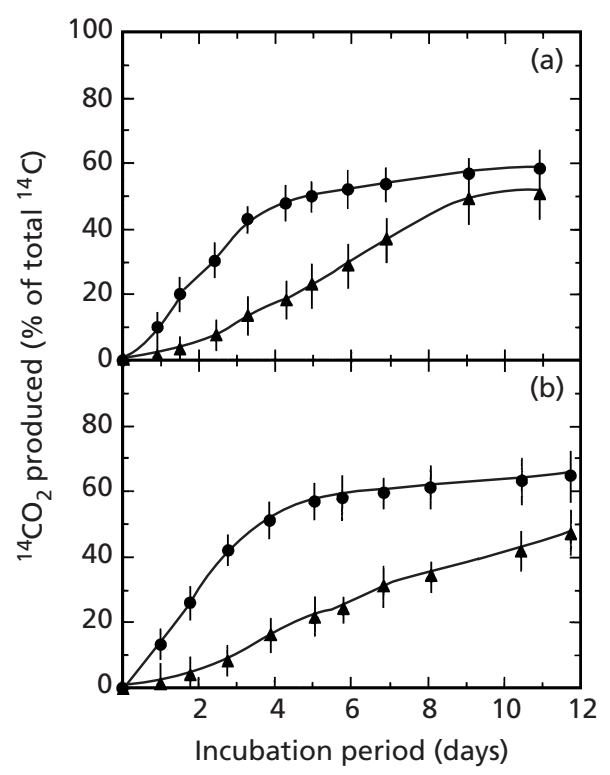

Fig. 5. Mineralization of benzaldehyde $(\boldsymbol{\Delta})$ and benzoic acid (O) by T. palustris (a) and G. trabeum (b). Each plot is the mean of the data obtained from three different cultures. The error bars show SD.

added to T. palustris cultures as substrates. They were effectively oxidized to the corresponding acid, and further metabolized (Fig. 3). Hydroquinone and trihydroxybenzene were metabolized but no formation of products was observed (Fig. 4).

It is also noteworthy that absolutely no activity of lignin and manganese peroxidases, or laccase, was observed at any stage of incubation. Nor was formation of coloured product, indicative of radical coupling reactions, observed when phenolic substrates were added to the cultures.

A comparison of substrate disappearance rates in $\mathrm{HN}$ and LN cultures showed that the rates were about twice as fast in the $\mathrm{HN}$ cultures, which was proportional to the dry weight of mycelia (data not shown). Nonspecific adsorption of the substrates was estimated by feeding them to azide-treated cells. The addition of all the substrates shown in Fig. 1 to cultures of the brownrot fungi, $10 \mathrm{~min}$ after the addition of $1 \mathrm{mM}$ sodium azide, resulted in the quantitative recovery of the substrates, indicating that non-specific adsorption of the substrates is not significant.

Recovery of aromatic compounds (remaining substrate plus aromatic intermediates) from the cultures either 4 or $48 \mathrm{~h}$ after substrate addition was determined. After $4 \mathrm{~h}$ incubation of benzaldehyde and benzoic acid with the brown-rot fungi, $85-95 \%$ recovery of aromatic components was observed; but after $48 \mathrm{~h}$ incubation, recovery fell to $35-55 \%$. These results strongly suggest the occurrence of ring fission by the brown-rot fungi. Purging with $100 \%$ oxygen ( $15 \mathrm{~min}$ per day) did not affect either the rate or the recovery for any of the substrates.

\section{Mineralization of aromatic compounds}

To further characterize the non-quantitative recovery of aromatic components (including quinones), ring-U- ${ }^{14} \mathrm{C}-$ labelled substrates were utilized. Fig. 5 shows the liberation of ${ }^{14} \mathrm{CO}_{2}$ from benzaldehyde and benzoic acid by $T$. palustris and G. trabeum, indicating the occurrence of the mineralization of aromatic components. $\mathrm{CO}_{2}$ liberation rates were somewhat faster with $G$. trabeum.

\section{Effect of hydroxyl radical scavengers on metabolism}

The addition of mannitol $(50 \mathrm{mM})$ or thiourea $(10 \mathrm{mM})$ to the culture media of $T$. palustris and G. trabeum during the metabolism of benzoic acid had no effect on either products or the rates of reactions (Table 2). The addition of catalase (5900 U) also had no effect on fungal conversion of benzoic acid. On the other hand, a Fentonreagent-driven conversion of benzoic acid to 4-hydroxybenzoic acid was completely inhibited by adding mannitol, thiourea or catalase (Table 2).

The effect of the cytochrome P450 inhibitors piperonyl butoxide (Franklin, 1972; Murray \& Reidy, 1990) and 1-aminobenzotriazole (Ortiz de Montellano \& Mathews, 1981) on the fungal degradation of benzoic acid was investigated. The effect of these inhibitos on fungal growth was minimal as indicated by the measurement of mycelial dry weight (data not shown). The absence of any inhibitory effect of P450 inhibitors on activities of fungal extracellular oxidases and ${ }^{14} \mathrm{CO}_{2}$ release from $\left[{ }^{14} \mathrm{C}\right]$ glucose was also reported (Eilers et al., 1999; Hiratsuka et al., 2001). Table 2 shows that the production of 4-hydroxybenzoic acid was partially, but not completely, inhibited by piperonyl butoxide and 1aminobenzotriazole. One possible explanation for incomplete inhibition is the difference of incorporation rates of benzoic acid and these inhibitors. Since benzaldehyde and benzoic acid were very rapidly metabolized by the fungi (Fig. 3), the incorporation of these compounds also seemed to be very effective. Then, the extracellular media of $T$. palustris after a 6 day incubation were exchanged with $500 \mathrm{mM}$ phosphate 
Table 2. Effect of hydroxyl radical scavengers, catalase and cytochrome $P 450$ inhibitors on the formation of 4-hydroxybenzoic acid (4-HBA) from benzoic acid via T. palustris metabolism and Fenton reaction

Experiments were done in triplicate.

\begin{tabular}{|c|c|c|}
\hline \multirow{2}{*}{$\begin{array}{l}\text { Treatment added to culture } \\
\text { or reaction mix }\end{array}$} & \multicolumn{2}{|c|}{ 4-HBA formed ( $\%$ of control $\pm \mathrm{SD}$ ) after: } \\
\hline & $\begin{array}{c}6 \mathrm{~h} \text { incubation of fungal } \\
\text { culture }\end{array}$ & $\begin{array}{c}24 \mathrm{~h} \text { incubation of Fenton } \\
\text { reaction }\end{array}$ \\
\hline Control & 100 & 100 \\
\hline $50 \mathrm{mM}$ mannitol & $102 \pm 4$ & 0 \\
\hline $10 \mathrm{mM}$ thiourea & $97 \pm 3$ & 0 \\
\hline $5900 \mathrm{U}$ catalase & $98 \pm 3$ & 0 \\
\hline $2.5 \mathrm{mM}$ piperonyl butoxide & $74 \pm 9$ & $96 \pm 3$ \\
\hline $2.5 \mathrm{mM}$ 1-aminobenzotriazole & $41 \pm 8$ & $101 \pm 4$ \\
\hline
\end{tabular}

buffer, $\mathrm{pH} 6 \cdot 0$. The mycelial mat was washed once with the buffer containing $50 \mathrm{mM}$ mannitol and $5 \mathrm{mM}$ EDTA, then twice with the buffer. The conversion of benzoic acid to 4-hydroxybenzoic acid occurred effectively after exchanging the extracellular medium $(1 \cdot 0-1 \cdot 3$ fold relative to the data shown in Fig. 3), indicating that the extracellular hydroxyl-radical-producing system is not involved in aromatic metabolism and that benzoic acid is very effectively incorporated into fungal cells.

\section{DISCUSSION}

The brown-rot fungi, which are taxonomically very similar to the white-rot basidiomycetes, are woodrotting fungi that decompose and preferentially remove carbohydrates, cellulose and hemicellulose. Bavendamm's reaction has indicated that the brown-rot fungi do not secrete phenoloxidases (Bavendamm, 1928; Kirk \& Kelman, 1965; Sundman \& Näse, 1971). Although laccase-like activity was recently found in G. trabeum cultures, the expressed level of the activity was reported to be 1000-fold less than that of the white-rot fungal control (D'souza et al., 1996). Furthermore, our data in this study clearly indicate that laccase does not play a role in the metabolism of phenolic and non-phenolic aromatics by brown-rot fungi.

\section{Metabolic pathway and mechanisms of benzaldehyde}

Benzaldehyde was mainly converted to benzyl alcohol and benzoic acid by T. palustris and G. trabeum (Figs 1-4). Although the conversion of benzaldehyde to alcohol was the main pathway (Fig. 2), benzyl alcohol was not further converted except for reoxidation back to benzaldehyde (data not shown). Thus, the oxidation of the formyl group to a carboxyl group seemed to be more important than the reduction during the metabolism. The reduction to form a hydroxymethyl group may be required to control the concentration of benzaldehyde in the fungal system because of its possible toxicity to the fungi. 4-Hydroxybenzaldehyde was converted to 4-hydroxybenzoic acid; on the other hand, no formation of 4-hydroxybenzaldehyde was observed from exogenously added 4-hydroxybenzoic acid, suggesting that the enzyme(s) involved in the conversion of benzaldehyde and benzoic acid may not catalyse that of 4-hydroxyl derivatives. 4-Hydroxybenzaldehyde and protocatechualdehyde seemed to be further metabolized via an intermediate formation of the corresponding acid (Fig. 3b, c).

Benzaldehyde and benzoic acid, especially benzoic acid, were effectively hydroxylated specifically at the C-4 position (Figs 1, 2 and 3a). Hydroxylation reactions were also involved in the formation of protocatechualdehyde and protocatechuic acid. From the present data, it is strongly suggested that the hydroxylation reactions play key roles in the metabolism of benzaldehyde by the two brown-rot fungi utilized in this study.

The hydroxylation reaction is very common and essential in the microbial degradation of aromatic compounds. The white-rot fungi cause aromatic hydroxylation through peroxidase-catalysed quinone formation followed by the action of quinone reductase (Brock \& Gold, 1996; Gold et al., 1989; Kirk \& Farrell, 1987; Tien, 1987; Valli \& Gold, 1991; Valli et al., 1992), as well as through the action of cytochrome P450s (Bezalel et al., 1996; Hiratsuka et al., 2001; Ichinose et al., 1999, 2002; Masaphy et al., 1996). It has also been reported that the hydroxyl radical might be formed by the brown-rot fungi during wood decay. A Fenton-type mechanism for hydroxyl radical generation by the brown-rot fungi has been proposed (Backa et al., 1992; Chandhoke et al., 1992; Goodell et al., 1997; Hirano et al., 1995, 1997; Hyde \& Wood, 1997; Kerem et al., 1998, 1999; Koenigs, 1975). In the present study, the addition of hydroxyl radical scavengers or catalase to cultures of brown-rot fungi during benzoic acid metabolism did not inhibit the production of 4-hydroxybenzoic acid (Table 2). However, this observation does not exclude the formation of a hydroxyl radical by brown-rot fungi as reported in previous studies. We assume that fungal production of a hydroxyl radical might be more influen- 


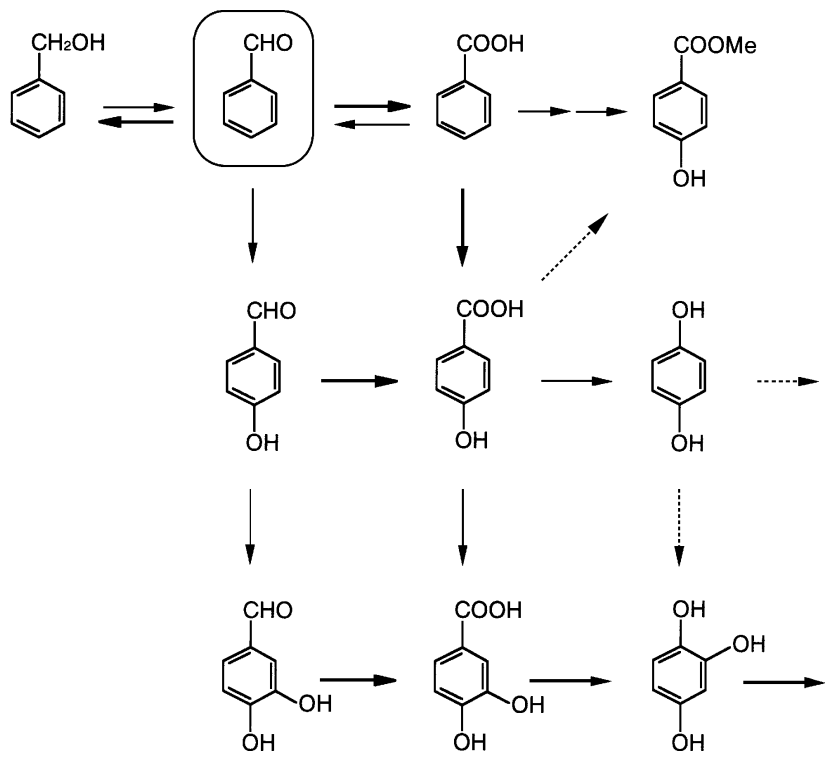

Fig. 6. Proposed metabolic pathway for benzaldehyde by brown-rot fungi.

tial for extracellular reactions, which seems to be advantageous for polymer degradation such as carbohydrates and polyethylene oxide, as previously reported (Hirano et al., 1995, 1997; Kerem et al., 1998). At this time, it is not possible to propose one definitive catalytic mechanism involved in the hydroxylation reactions observed in this study. However, the peroxidasecatalysed quinone formation can at least be eliminated, since no activities of peroxidalaccases were observed. Our preliminary observations indicated that cytochrome P450 inhibitors showed some inhibitory effect (Table 2). The involvement of intracellular cytochrome-P450mediated hydroxylation reactions in the metabolism of monomeric aromatic compounds by brown-rot fungi needs to be investigated. Identification of cytochrome P450 in brown-rot fungi is now being attempted.

Both brown-rot fungi yielded almost identical products from benzaldehyde. Methylation of 4-hydroxybenzoic acid was only seen with T. palustris. Except for this methylation reaction, both fungi metabolize benzaldehyde by the same mechanism. The rate of benzaldehyde metabolism was twice as fast with G. trabeum (data not shown). That is partly because G. trabeum did not accumulate the dead-end product methyl 4-hydroxybenzoate, whereas T. palustris did so.

From the comparison of disappearance rates shown in Fig. 4, hydroquinone seemed to be converted to trihydroxybenzene and further degraded. The low level of accumulation of trihydroxybenzene during benzaldehyde metabolism (Fig. 2) suggested that this compound might be a direct substrate for the ring-fission reaction. The occurrence of the ring-fission reaction was proved by ${ }^{14} \mathrm{C}$-labelling experiments (Fig. 5).

All these data allow us to propose the reaction pathways of benzaldehyde by the brown-rot fungi (Fig. 6). The oxidation and reduction at the benzylic position occur simultaneously, forming benzyl alcohol and benzoic acid as major products. Hydroxylation reactions, which seem to be a key step, occur on benzaldehyde and benzoic acid, but not on benzyl alcohol, to form corresponding 4-hydroxyl and 3,4-dihydroxyl derivatives. 1-Formyl derivatives are oxidized to 1-carboxyl derivatives at several metabolic stages. All these reactions result in the formation of 3,4-dihydroxybenzoic acid. This is further metabolized via the decarboxylation reaction to yield 1,2,4-trihydroxybenzene, which may be susceptible to the ring-fission reaction. It may be too early to make broad generalizations about the reaction mechanism of the brown-rot fungi; however, at least the two fungi used in this study share the same mechanism.

\section{ACKNOWLEDGEMENTS}

This research was supported by a Grant-in-Aid for Scientific Research from the Japan Society for Promotion of Science and from the New Energy and Industrial Technology Development Organization (NEDO) of Japan (to H.W.).

\section{REFERENCES}

Backa, S., Gierer, J. \& Reitberger, T. (1992). Hydroxyl radical activity by brown-rot fungi studied with a new chemiluminescence method. Holzforschung 46, 61-67.

Bavendamm, W. (1928). Originalabhandlungen. Über das Vorkommen und den Nachweis von Oxydasen bei Holzzerstörenden Pilzen. Z Pflanzenkr Pflanzenschuz 38, 257-276.

Bezalel, L., Hadar, Y., Fu, P. P., Freeman, J. P. \& Cerniglia, C. (1996). Initial oxidation products in the metabolism of pyrene, anthracene, fluorene, and dibenzothiophene by the white rot fungus Pleurotus ostreatus. Appl Environ Microbiol 62, 25542559.

Brock, B. J. \& Gold, M. H. (1996). 1,4-Benzoquinone reductase from the basidiomycete Phanerochaete chrysosporium: spectral and kinetic analysis. Arch Biochem Biophys 331, 31-40.

Brown, W., Cowling, E. B. \& Falkehag, S. I. (1968). Molecular size distributions of lignins liberated enzymatically from wood. Sven Papperstidning 22, 811-821.

Chandhoke, V., Goodell, B., Jellison, J. \& Fekete, F. A. (1992). Oxidation of 2-keto-4-thiomethylbutyric acid (KTBA) by ironbinding compounds produced by the wood-decaying fungus Gloeophyllum trabeum. FEMS Microbiol Lett 90, 263-266.

Crawford, R. L. (1981). Lignin Biodegradation and Transformation. New York: Wiley-Interscience.

D'souza, T. M., Boominathan, K. \& Reddy, C. A. (1996). Isolation of laccase gene-specific sequences from white rot and brown rot fungi by PCR. Appl Environ Microbiol 62, 3739-3744.

Eilers, A., Rüngeling, E., Stündl, U. M. \& Gottschalk, G. (1999). Metabolism of 2,4,6-trinitrotoluene by the white-rot fungus Bjerkandera adusta DSM 3375 depends on cytochrome P-450. Appl Microbiol Biotechnol 53, 75-80.

Enoki, A., Takahashi, M., Tanaka, H. \& Fuse, G. (1985). Degradation of lignin-related and wood components by white-rot and brown-rot fungi. Mokuzai Gakkaishi 31, 397-408.

Eriksson, K.-E. L., Blanchette, R. A. \& Ander, P. (1990). Microbial and Enzymatic Degradation of Wood and Wood Components. Berlin: Springer.

Franklin, M. R. (1972). Inhibition of hepatic oxidative xenobiotic 
metabolism by piperonyl butoxide. Biochem Pharmacol 21, 3287-3299.

Gold, M. H., Wariishi, H. \& Valli, K. (1989). Extracellular peroxidases involved in lignin degradation by the white rot basidiomycete Phanerochaete chrysosporium. In Biocatalysis in Agricultural Biotechnology (ACS Symposium Series vol. 389), pp. 127-140. Edited by J. R. Whitaker. \& P. E. Sonnet. Washington DC: American Chemical Society.

Goodell, B., Jellison, J., Liu, J., Daniel, G., Paszczynski, A., Fekete, F., Krishnamurthy, S., Jun, L. \& Xu, G. (1997). Low molecular weight chelators and phenolic compounds isolated from wood decay fungi and their role in the fungal biodegradation of wood. J Biotechnol 53, 133-162.

Hirano, T., Tanaka, H. \& Enoki, A. (1995). Extracellular substance from the brown-rot basidiomycete Tyromyces palustris that reduces molecular oxygen to hydroxyl radicals and ferric iron to ferrous iron. Mokuzai Gakkaishi 41, 334-341.

Hirano, T., Tanaka, H. \& Enoki, A. (1997). Relationship between production of hydroxyl radicals and degradation of wood by the brown-rot fungus, Tyromyces palustris. Holzforschung 51, 389395.

Hiratsuka, N., Wariishi, H. \& Tanaka, H. (2001). Degradation of diphenyl ether herbicides by the lignin-degrading basidiomycete Coriolus versicolor. Appl Microbiol Biotechnol 57, 563-571.

Hyde, S. M. \& Wood, P. (1997). A mechanism for production of hydroxyl radicals by the brown-rot fungus Coniophora puteana: $\mathrm{Fe}(\mathrm{III})$ reduction by cellobiose dehydrogenase and Fe(II) oxidation at a distance from the hyphae. Microbiology 143, 259-266.

Ichinose, H., Wariishi, H. \& Tanaka, H. (1999). Bioconversion of recalcitrant 4-methyldibenzothiophene to water-extractable products using lignin-degrading basidiomycete Coriolus versicolor. Biotechnol Prog 15, 706-714.

Ichinose, H., Wariishi, H. \& Tanaka, H. (2002). Identification and characterization of novel cytochrome $\mathrm{P} 450$ genes from the whiterot fungus Coriolus versicolor. Appl Microbiol Biotechnol 58, 97-105.

Kerem, Z., Bao, W. \& Hammel, K. (1998). Rapid polyether cleavage via extracellular one-electron oxidation by a brown-rot basidiomycete. Proc Natl Acad Sci U S A 95, 10373-10377.

Kerem, Z., Jensen, K. A. \& Hammel, K. (1999). Biodegradative mechanism of the brown rot basidiomycete Gloeophyllum trabeum: evidence for an extracellular hydroquinone-driven Fenton reaction. FEBS Lett 446, 49-54.

Kirk, T. K. (1975). Effects of a brown-rot fungus, Lenzites trabea, on lignin in spruce wood. Holzforschung 29, 99-107.

Kirk, T. K. \& Farrell, R. L. (1987). Enzymatic "combustion": the microbial degradation of lignin. Annu Rev Microbiol 41, 465-505.
Kirk, T. K. \& Kelman, A. (1965). Lignin degradation as related to the phenoloxidases of selected wood-decaying basidiomycetes. Phytopathology 55, 739-745.

Kirk, T. K., Schultz, E., Connors, W. J., Lorenz, L. F. \& Zeikus, J. G. (1978). Influence of culture parameters on lignin metabolism by Phanerochaete chrysosporium. Arch Microbiol 117, 277-285.

Koenigs, J. W. (1975). Hydrogen peroxide and iron: a microbial cellulolytic system. Biotechnol Bioeng Symp 5, 151-159.

Masaphy, S., Levanon, D., Henis, Y., Venkateswarlu, K. \& Kelly, S. L. (1996). Microsomal and cytosolic cytochrome P450 mediated benzo(a)pyrene hydroxylation in Pleurotus pulmonarius. Biotechnol Lett 17, 967-974.

Murray, M. \& Reidy, G. F. (1990). Selectivity in the inhibition of mammalian cytochromes P-450 by chemical agents. Pharmacol Rev 42, 85-101.

Ortiz de Montellano, P. R. \& Mathews, J. M. (1981). Autocatalytic alkylation of the cytochrome P-450 prosthetic haem group by 1aminobenzotriazole. Isolation of an NN-bridged benzyne-protoporphyrin IX adduct. Biochem J 195, 761-764.

Paszczynski, A., Crawford, R., Funk, D. \& Goodell, B. (1999). De novo synthesis of 4,5-dimethoxycatechol and 2,5-dimethoxyhydroquinone by the brown rot fungus Gloeophyllum trabeum. Appl Environ Microbiol 65, 674-679.

Sundman, V. \& Näse, L. (1971). A simple plate test for direct visualization of biological lignin degradation. Papperi ja Puи 53, $67-71$.

Tien, M. (1987). Properties of ligninase from Phanerochaete chrysosporium and their possible applications. CRC Crit Rev Microbiol 15, 141-168.

Valli, K. \& Gold, M. H. (1991). Degradation of 2,4-dichlorophenol by the lignin-degrading fungus Phanerochaete chrysosporium. J Bacteriol 173, 345-352.

Valli, K., Wariishi, H. \& Gold, M. H. (1992). Degradation of 2,7dichlorodibenzo- $p$-dioxin by the white-rot basidiomycete, $\mathrm{Pha}$ nerochaete chrysosporium. J Bacteriol 174, 2131-2137.

Wariishi, H., Valli, K. \& Gold, M. H. (1989). Oxidative cleavage of a phenolic diarylpropane lignin model dimer by manganese peroxidase from Phanerochaete chrysosporium. Biochemistry 28, 6017-6023.

Wariishi, H., Valli, K. \& Gold, M. H. (1992). Manganese(II) oxidation by manganese peroxidase from the basidiomycete Phanerochaete chrysosporium: kinetic mechanism and role of chelators. J Biol Chem 267, 23688-23695.

Zabel, R. A. \& Morrell, J. J. (1992). Wood Microbiology: Decay and its Prevention. San Diego: Academic Press.

Received 30 November 2001; revised 18 February 2002; accepted 7 March 2002. 